\title{
Supervisión pedagógica y gestión administrativa de las tic en el aip del CEBA de Cusco
}

\author{
Pedagogical supervision and administrative management of ict in the aip of the CEBA of Cusco
}

Supervisão pedagógica e gestão administrativa de ict na aip do CEBA de Cusco

\section{ARTÍCULO GENERAL}

\section{Abigail Chávez Meza \\ https://orcid.org/0000-0002-9233-0220 \\ chmeza45@gmail.com}

Pontificia Universidad Católica del Perú, Lima - Perú

Recibido 02 de Octubre 2021 | Arbitrado y aceptado 14 de Diciembre 2021 | Publicado en 04 Marzo 2022

\section{RESUMEN}

La presente investigación es una aproximación teórica al estudio de la supervisión pedagógica y gestión administrativa de las TIC en aulas de innovación pedagógica de un Centro Educativo Básico Alternativo del Cusco. Para entender esta situación, tomamos como punto de partida, el diagnóstico de la gestión de un CEBA, del Cusco con el propósito de conocer lo que se viene trabajando en materia de supervisión y gestión administrativa de las TIC en la institución en estudio. Esta investigación busca profundizar el estudio de la supervisión y gestión administrativa de las TIC en el CEBA piloto y elaborar estrategias orientadas al establecimiento de un sistema de las TIC, considerando las corrientes actuales de supervisión y gestión administrativa.

Las conclusiones a la que arribamos es sque las acciones de supervisión, monitoreo y acompañamiento se cumple de manera regular, siendo fundamental la aplicación de una política educativa integral que logre los propósitos nacionales en educación.

PALABRAS CLAVE: Supervisión pedagógica, Gestión administrativa de las TIC.

\section{ABSTRACT}

This research is a theoretical approach to the study of the pedagogical supervision and administrative management of ICT in pedagogical innovation classrooms of an Alternative Basic Educational Center of Cusco. To understand this situation, we take as a starting point, the diagnosis of the management of a CEBA, of Cusco in order to know what is being worked on in terms of supervision and administrative management of ICT in the institution under study.

This research seeks to deepen the study of the supervision and administrative management of ICT in the pilot CEBA and to develop strategies aimed at the establishment of an ICT system, considering the current supervision and administrative management flows.

The conclusions we reached is that the actions of supervision, monitoring and accompaniment are carried out on a regular basis, being fundamental the application of a comprehensive educational policy that achieves national purposes in education.

KEY WORDS: $\begin{array}{r}\text { Pedagogical } \\ \text { supervision, }\end{array}$
Administrative management of ICT.

\section{RESUMO}

Esta pesquisa é uma abordagem teórica para o estudo da supervisão pedagógica e gestão administrativa das TIC em salas de aula de inovação pedagógica de um Centro Educacional Básico Alternativo de Cusco. Para compreender esta situação, partimos do diagnóstico da gestão de um CEBA, de Cusco, para saber o que se está a trabalhar em matéria de supervisão e gestão administrativa das TIC na instituição em estudo.

Esta pesquisa visa aprofundar o estudo da supervisão e gestão administrativa das TIC no CEBA piloto e desenvolver estratégias voltadas ao estabelecimento de um sistema de TIC, considerando os atuais fluxos de supervisão e gestão administrativa.

A conclusão a que se chegou é que as ações de supervisão, acompanhamento e acompanhamento são realizadas de forma regular, sendo fundamental a aplicação de uma política educacional integral que alcance objetivos nacionais na educação.

PALAVRAS-CHAVE: Supervisão pedagógica, Gestão administrativa das TIC. 


\section{1.-INTRODUCCIÓN:}

La siguiente pregunta de investigación:

La presente investigación pretende profundizar el ¿De qué manera se relaciona la Supervisión estudio de la Supervisión pedagógica y de gestión Pedagógica y la Gestión administrativa de las TIC administrativa de las TIC en los Centros Básicos en las aulas de Innovación Pedagógica de un

Centro Educativo Básico Alternativo CEBA Piloto de la Ciudad del Cusco?

Se ha planteado como objetivo general y sus respectivos objetivos específicos. Determinar de qué manera se relaciona la Supervisión Pedagógica y la gestión administrativa de las TIC, en las aulas de innovación pedagógica, perteneciente a un Centro Educativo Básico Alternativo CEBA Piloto de la Ciudad del Cusco. Y como objetivos específicos.

Determinar si el monitoreo en la Supervisión pedagógica se relaciona con la gestión administrativa de las TIC en las aulas de Innovación Pedagógica del Centro Educativo Básico Alternativo CEBA Piloto de la Ciudad del Cusco. Establecer si el acompañamiento se relaciona con la Supervisión pedagógica y la Gestión administrativa de las TIC y el marco de referencia de las aulas de Innovación Pedagógica del CEBA. Analizar si la gestión administrativa se relaciona en la Supervisión Pedagógica con la gestión administrativa de las TIC en el aula de Innovación pedagógica del CEBA.

En cuanto al diseño de investigación corresponde al tipo no experimental, el cual según Hernández, et. al (2010) no implica una modificación o producción del fenómeno a investigar sino que se limita a descubrir una situación o determinadas características del objeto, dirigida al estudio cuantitativo.

La institución donde se realizó el estudio está constituida por un CEBA Piloto situado en la ciudad del Cusco la cuál atienden a una población de estudiantes a partir de los 14 años en adelante.

Para el recojo de datos, se utilizó como instrumento la encuesta. Para la misma, se utilizó como instrumento un cuestionario de likert, que nos permitió recoger información a través de la encuesta, dirigido a tres tipos de sujetos (Suárez, 2003)

En el presente estudio, se tomó en consideración a tres sujetos (director del CEBA, docentes del ciclo avanzado y alumnos de diferentes secciones del ciclo avanzado 1ro al 
4to ciclo. Con ello se ha pretendido tener la más completa y precisa información por parte de los informantes.

El instrumento arriba mencionado, ha sido validado por un grupo de expertos. Posteriormente, la guía de encuesta se aplicó a la muestra seleccionada y al procesamiento de los resultados utilizando la técnica respectiva de tratamiento de información luego se extrajo el resultado correspondiente.

Las conclusiones a la que hemos arribado es que las acciones de supervisión, monitoreo y acompañamiento se cumple de manera regular, siendo fundamental la aplicación de una política educativa integral que permita lograr los propósitos nacionales a nivel de educación, así mismo la correlación estadística es de nivel regular respecto al proceso de agilizar procesos administrativos con el apoyo de las TIC.

Así mismo el aporte de esta investigación es conocer de manera científica el desarrollo de una adecuada Supervisión pedagógica y la gestión administrativa de las TIC que pueda servir para un mejor seguimiento a las políticas TIC en el Perú y por ende a las aulas de innovación pedagógica de los Centros Educativos Básicos alternativos CEBA de la ciudad del Cusco.

\section{2.- La Supervisión Educativa en el Sistema Educativo Peruano}

Muchos son los estudiosos de esta especialidad pedagógica y diversos los conceptos que cada uno de ellos tiene sobre lo que se entiende por supervisión educativa, una síntesis apretada, nos refiere que esta actividad es un servicio instituido para optimizar el funcionamiento de las organizaciones educativas, centrado en el mejoramiento de las condiciones de aprendizaje, referente al desenvolvimiento profesional de los maestros y el trabajo curricular desarrollado en el aula. A continuación se describe algunas definiciones dadas por algunos autores:

a) Para el Ministerio de Educación del Perú, La Supervisión pedagógica es un proceso intencionado y sistemático de carácter técnico de orientación, asesoramiento instituido. Para optimizar las actividades pedagógicas en las instancias de la gestión educativa descentralizada. Está centrada en el proceso continuo de los procesos de aprendizaje, el desempeño profesional de los docentes y el ofrecimiento de la oportuna y consistente información, para una acertada toma de decisiones.

Respecto a la finalidad, tomando en cuenta que la finalidad es el propósito o razón de ser de una meta trazada. Actualmente se indica que la finalidad de la supervisión es la mejora del Sistema Educativo en todos sus aspectos, implicando con ello lo institucional, lo 
administrativo, y lo pedagógico, más la supervisión pondrá especial énfasis en los aspectos pedagógicos.

Se concluye que la supervisión pedagógica es un servicio técnico experto no una actividad de inspección y marcada autoridad sobre el personal supervisado, por lo tanto ayuda, aporta, comparte y contribuye al proceso enseñanza- aprendizaje, mediante un conjunto organizado de principios, métodos, técnicas efectivas y pertinentes, calificada como un servicio de asesoramiento de todas las actividades que influyen en el proceso de enseñanza aprendizaje con miras a realizar un mejor planeamiento una mejor coordinación y ejecución de las mismas, para que se atienda en forma eficiente a las necesidades y aspiraciones del alumno y la comunidad educativa así como para que se lleven a efecto más plenamente los objetivos generales de la educación y los objetivos específicos de la escuela. (MINEDU, 2009)

a)Funciones de la Supervisión Educativa, en el contexto educativo del siglo XXI la supervisión aparece ampliamente impregnada, por la concepción de lo que conocemos como acompañamiento pedagógico en tanto "acción de asesoramiento" o por la concepción de monitoreo, que supone acciones de recojo sistemático de información", sin embargo no son conceptos de la misma jerarquía, sino tanto el acompañamiento como el monitoreo, son funciones de la supervisión pedagógica que al igual que la función de control, se ejercen en forma sincrónica y de acuerdo a objetivos previamente determinados. Como son: La Función de Control, función administrativa de la supervisión caracterizada por el proceso de verificación del cumplimiento de la normatividad que rige para las entidades educativas y las funciones de los profesionales de educación, orientado a garantizar el desarrollo del año escolar. (MINEDU, 2009) -Función de Monitoreo, función que consiste en el recojo, análisis y procesamiento de datos que interpretados se convierten en información útil para la toma de decisiones, oportunas, pertinentes de carácter técnico para la mejora de los procesos a nivel de aula -Función de Acompañamiento, es la función pedagógica de supervisión orientada a fortalecer el desempeño profesional del docente por la vía de asistencia técnica, basada en el intercambio de experiencias entre el acompañante y acompañado.

a) Objetivos de la Supervisión Educativa, se presenta los siguientes:

-Objetivos Generales, en congruencia con el Plan estratégico sectorial multianual se asumen los siguientes objetivos: 
-Incrementar niveles de calidad y equidad a los servicios del sector educación.

-Promover el conocimiento, la práctica de la ciencia, tecnología, cultura y deporte.

-Asegurar la implementación de las políticas del sector en los gobiernos regionales y locales con participación de la sociedad.

-Objetivos Específicos:

-Recabar la información relevante y actualizada sobre la gestión educativa y sus resultados integrar de manera coherente los datos de los diversos contextos educativos.

-Establecer mecanismos que ayuden y estimulen a los agentes educativos para mejorar su intervención en función de los estándares educativos nacionales a través de su involucramiento responsable.

Tomar decisiones estratégicas en todas las instancias de gestión educativa descentralizada orientadas a mejorar la calidad de la supervisión educativa, sobre la base integral de los problemas, resultados e impactos de la labor educativa (MINEDU 2009)

Principios de la Supervisión Educativa, la supervisión se sustenta en los principios de la educación peruana: ética, inclusión calidad, democracia, interculturalidad, conciencia ambiental, creatividad, e innovación (Artículo 8 de la Ley general No 28044), sobre esta base se plantean principios particulares de la supervisión pedagógica que regulan y dan unidad a las prácticas supervisoras, con un enfoque de desarrollo humano, se distingue entre esto principios: -Principio de Cooperación La supervisión pedagógica, debe desenvolverse con un espíritu de colaboración entre el supervisor y el supervisado y los demás agentes que contribuyen al logro de los aprendizajes previstos.

-Principio de Concertación, la supervisión pedagógica debe promover el espíritu de participación y concertación sobre todo en la toma de decisiones estratégicas en todas las instancias de la gestión educativa descentralizada.

-Principio de Oportunidad, la supervisión pedagógica debe constituirse en una actividad permanente del proceso educativo que permita identificar, anticipar las necesidades específicas y brindar con oportunidad estrategias y contenidos diferenciados así como garantizar el logro del plan de acción.

-Principio de Objetividad, la supervisión pedagógica debe perseguir la objetividad, lo que amerita planear y desarrollar algún tipo de investigación científica

de manera que el conocimiento y la información producida se fundamente en hechos comprobados y no suposiciones de corte subjetivo y empírico.

-Principio de Practicidad, la supervisión pedagógica se concretiza en el campo, por lo tanto se debe privilegiar la observación directa de los procesos pedagógicos en el 
hecho educativo y verificar si el docente está enseñando lo que tiene que enseñar, y si el alumno está aprendiendo lo que tiene que aprender y saber hacer de acuerdo a los estándares nacionales, regionales y locales.

\section{3.-La Supervisión del tratamiento de las TIC en los Centros Educativos.}

El desarrollo de las competencias relacionadas con las Tecnologías de la Información y Comunicación (TIC), se encuentra presente en todas las etapas de nuestro sistema educativo. Por ello en el marco de la Supervisión que corresponde a la inspección de educación, se hace necesario disponer una herramienta que oriente la intervención para comprobar el tratamiento de las TIC en los centros Educativos con especial incidencia en la práctica docente. Este artículo ofrece una propuesta de elementos y procesos a supervisar en relación con la utilización de las TIC en el ámbito educativo.

Las tecnologías de la información y la comunicación (TIC) (Cornax, 2006), se encuentran plenamente integradas en nuestra sociedad, lo que supone cambios importantes en el enfoque que se ha de dar a la enseñanza e incluso en lo que es objeto de aprendizaje. Por ello, las diferentes administraciones educativas han hecho importantes esfuerzos económicos para acercar las TIC al alumnado. El enfoque que se le ha dado a las TIC en el ámbito educativo ha sido muy diverso, e incluso, podríamos afirmar que poco estructurado en la práctica. Con este artículo pretendemos ofrecer un modelo de supervisión educativa enfocado a un adecuado tratamiento de las TIC en los centros educativos.

Por otra parte la irrupción de las TIC afecta a la esfera de la enseñanza, por lo que los docentes se enfrentan a retos, entre los que están el uso técnico de las TIC y la selección y transformación de la información, la utilización de las TIC como herramientas de aprendizaje y comunicación y comprensión crítica de las informaciones que aportan las TIC.

Por tanto la idea de Supervisión de las TIC en un centro Educativo que vamos a desarrollar se basa en el concepto de competencia TIC, esto va enfocada que debe ir dirigida a que sus alumnos y alumnas sean competentes en el uso de las tecnologías de la información y comunicación. Este alumnado no sólo ha de ser capaz de usar y configurar diferentes medios tecnológicos y su correspondiente software, sino que además debe ser capaz de usar y configurar diferentes medios tecnológicos y su correspondiente software, sino que 
además debe ser capaz de reflexionar sobre las implicaciones que la presencia de esos medios tiene en la esfera social, laboral, personal.

\section{4.-Uso de las TIC en la Administración.}

Ciertamente las TIC son percibidas como herramientas, pero no se ha llegado a realizar el análisis sobre el mismo como medio para detectar y valorar situaciones, que pueden ser de mucho aporte en la toma de decisiones, sobre todo en el sector educativo.

Las Instituciones Educativas que pretenden ser comunidades de aprendizaje, requieren entrenarse en la reflexión sobre las acciones para poder construir aprendizajes que, por ende influirán en la toma de decisiones. Para los orientales, el aprendizaje implica el estudio y la práctica constante, incluso se sugiere la idea de que el verdadero aprendizaje está ligado al autoaprendizaje que deriva de la reflexión (Senge,

1993 p.10)

Desde este punto de vista se debe gestionar el uso de las TIC en el contexto administrativo, donde todos están comprometidos y participan activamente, siendo la tecnología "herramienta para y soporte del proceso para". Es así que la cultura organizacional desde este punto asume un rol importante y los valores se convierten en un eje de acción.

Para determinar el tipo de tecnología que pueda beneficiar la organización es necesario tener en cuenta algunos criterios: -El Software debe ser adaptable

-La tecnología debe ser flexible

-El hardware y software son escalables -La soluciones de software y hardware no deben ser costosas.

5.- Los Centros Educativos Básicos Alternativos y el uso de aulas de Innovación Pedagógica según normas vigentes del Perú.

La Ley General de Educ ación reconoce que la educación es un derecho fundamental de todos, brindando calidad que asegure condiciones adecuadas para una educación integral, pertinente, abierta, flexible, inclusiva y permanente.

DCNB- MED 2009.

La Ley General de Educación, en un esfuerzo de transformación estructural del sistema educativo nacional, institucionaliza la Educación Básica Alternativa -EBA- como una modalidad de la Etapa de Educación Básica, equivalente a la Educación Básica Regular 
en calidad y logros de aprendizajes enfatiza la preparación para el trabajo y el desarrollo de capacidades empresariales de los estudiantes. La EBA está orientada a niños y adolescentes en extraedad, así como a jóvenes y adultos, que por diversas razones no tuvieron acceso a la Educación Básica Regular o no pudieron culminarla, y para aquellos que necesitan compatibilizar el trabajo con el estudio.

\begin{tabular}{|l|l|l|l|}
\hline \multicolumn{3}{|c|}{ EDUCACIÓN BÁSICA ALTERNATIVA } \\
\hline \multirow{4}{*}{ PROGRAMAS } & CICLO & CICLO & CICLO \\
& INICIAL & INTERMEDIO & AVANZADO. \\
& $\begin{array}{l}\text { PEBANA, } \\
\text { PEBAJA. }\end{array}$ & PEBAJA & PEBAJA \\
FORMAS DE & Presencial. & PEBANA & PEBANA \\
ATENCIÓN & Semipresencial & Semipresencial \\
& & A distancia. & A distancia. \\
\hline
\end{tabular}

Tabla N 4: Elaboración Propia, tomada de DCNB -2009 MINEDU.

Aulas de Innovación Pedagógica

Tabla $\mathrm{N}^{\circ} 05$ DCNB-MED 2009.

\section{6.-Teorías que sustenten el uso de}

los estudiantes en el sistema educativo formal, de darles una educación compensatoria. Desde una concepción más amplia de la EBA, supone la satisfacción de las necesidades básicas de aprendizaje que requiere todo ser humano para su desarrollo, ejercer ciudadanía y continuar aprendiendo autónomamente durante toda la vida.
1.-Organización
de
la
Educación
Básica 
Alternativa

2.-La EBA se organiza en programas y en ciclos que se ofrecen en tres formas de atención. Esto se puede visualizar en la siguiente tabla:

del ecosistema comunicativo sustentado principalmente por Barbero. Estos tres enfoques confluyen en una mirada triangular desde las perspectivas psicológicas, educativas y comunicativas, que favorecen el desarrollo de las capacidades TIC en el ámbito educativo.

\section{a) Enfoque Socio-Cultural}

El enfoque sociocultural es clave y fundamental abordarlo cuando en el Aprendizaje.

En este trabajo de investigación hemos considerado abordar sobre los diferentes enfoques relacionados con el desarrollo de capacidades TIC. (Conde et al 2012), es importante señalar que hay teorías que sustentan cómo se desarrollan las capacidades TIC, en el nuevo contexto socio tecnológico que nos encontramos y que consideramos valioso abordarlo porque hay una enorme potencialidad de desarrollar las capacidades en el ámbito educativo, a través de procesos de enseñanza aprendizaje. En este marco, de acuerdo, a la amplia investigación que tenemos sobre el tema, consideramos pertinente, abordar tres enfoques: enfoque sociocultural cuyo principal gestor es Vigotsky, el enfoque de las cogniciones distribuidas sustentado por Salomon y el enfoque estamos hablando sobre el desarrollo de capacidades en las aulas de innovación, puesto que el aprendizaje bajo esta concepción se entiende como social, es decir, un aprendizaje en interacción con otras personas y mediadas por instrumentos culturales que son las herramientas y los signos. Vigotsky, propone la necesidad de instrumentos de mediación que faciliten la interacción entre el sujeto y el objeto de mediación. (Choque, 2009, p. 126)

Desde esta perspectiva podemos considerar a la computadora e Internet, como aquellos que mediatizan las relaciones como si las personas estarían cara a cara. La postura Vigotskyana presupone que la mente surge de la actividad mediada conjuntamente entre las personas y por ende la mente es construida y distribuida. 
b) Enfoque de la Cognición Distribuida Las cogniciones distribuidas son un enfoque que nos permite clarificar con mucha precisión el cómo se van desarrollando las capacidades en las aulas de innovación, a través de procesos cognitivos que se generan al utilizar las los elementos que componen las aulas de innovación como es el caso de Internet. Cuando hablamos de cogniciones distribuidas, partimos del hecho de que los seres humanos no aprendemos solos, sino aprendemos de un conjunto, de una red de situaciones donde los instrumentos son sumamente importantes. Y actualmente en la Sociedad Red, contamos con una serie de instrumentos que favorecen la interacción de las personas, así como el almacenamiento, organización y difusión de información

Por lo tanto un reto fundamental es que en la educación se debería alcanzar hacia una inteligencia distribuida de manera reflexiva e intencional, donde los estudiantes sean los inventores de inteligencia distribuida como una herramienta y no receptores de inteligencia como sustancia. Un aspecto central que debemos considerar es que las aulas de innovación no son solamente un simple medio, sino son artefactos culturales que permite reorganizar el funcionamiento mental de las personas. En esta perspectiva es clave que el sistema educativo tome en cuenta este aspecto e integre las aulas de innovación de una manera técnica.

c) Enfoque del Ecosistema Comunicativo En el campo de la educación, se viene generando una ruptura con lo que es la organización y enseñanza tradicional, donde está surgiendo un nuevo espacio didáctico como señala Pérez (2000, p. 25), “donde se perfila un imprescindible proceso de reconversión docente estrechamente ligado a los cambios que se van originando en nuestra sociedad conforme se va generalizando el uso de las nuevas tecnologías".

El ciberespacio se configura aquí como nuevo contexto educativo, así gracias a estos recursos y herramientas los procesos de enseñanzaaprendizaje pueden ser desarrollados en diversos contextos: la institución educativa, el domicilio del estudiante, el lugar de trabajo. En relación a los contenidos educativos en el ecosistema comunicativo, el Internet se ha configurado como una fuente amplia y heterogénea de información donde se puede encontrar casi todos los temas, pues como refiere Pérez "Internet es hoy posiblemente el mayor depositario de información disponible que hay en el mundo" (Pérez 2000, p. 170). Hasta hace poco era impensable conseguir, pero ahora gracias al Internet uno consigue información de cualquier parte del mundo y sobre cualquier tema. En el campo educativo se viene configurando una serie de accesos a bibliotecas 
digitales, diccionarios, centros de investigación, centros de documentación, etc. Sobre el tema de la metodología educativa, la adaptación curricular paulatina a las nuevas tecnologías y especialmente a Internet, conlleva un cambio metodológico puesto que en la enseñanza tradicional las fuentes de información en el ámbito educativo eran el libro de texto y el profesor.

7.-Metodología de la Investigación

Para el desarrollo de la investigación se ha recurrido a los siguientes métodos:

a. Deductivo - Sintético: Se utilizó este método para revisar e identificar la teoría que sustente las hipótesis planteadas y luego desglosarlas en conceptos más pequeños y concretos que permitan medir las características de ambas variables.

b. Crítico - Comparativo: Este método fue utilizado para discutir y comparar los resultados obtenidos, producto del procesamiento estadístico y las conclusiones obtenidas por otras investigaciones. Asimismo, fue utilizado para analizar y seleccionar las fichas bibliográficas que pasaron a conformar el contenido del marco teórico.

\section{1.- Población y muestra}

La población estará conformada por el CEBA Inca Garcilaso de la Vega, teniendo nivel y modalidad Básico Alternativa Avanzada, con 268 alumnos, 29 docentes, se hicieron 23 (por motivos, personales, permisos por salud y otros) 1 Director.

La muestra es una parte o subconjunto de una población normalmente seleccionada de tal modo que pone de manifiesto las propiedades de la población. Su característica más importante es la representatividad, es decir, que sea una parte típica de la población en las características que son relevantes para la investigación.

En este estudio, la muestra es aleatoria simple. 
$n$

$$
=\frac{(p * q) * \mathrm{Z}^{2} * N}{\mathrm{E}^{2}(\mathrm{~N}-1)+(\mathrm{p} * \mathrm{q}) \mathrm{Z}^{2}}
$$

Donde:

$\mathrm{n}$

$=$ Muestra .

$\mathrm{N}=$ Población

268

$Z=$ Nivel de confianza $95 \%$

$1.96 \quad \mathrm{e} \quad$ Error

$5 \%$

$0,05 \quad \mathrm{p}=$ Probabilidad de éxito

$50 \%$

0,5 $\mathrm{q}=$ Probabilidad de fracaso

$50 \%$

$$
0,5
$$

Reemplazando, tenemos:

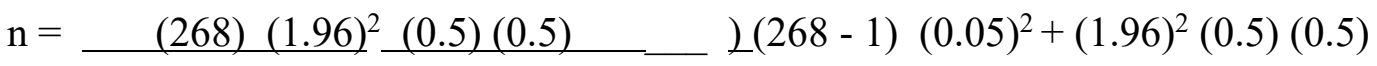

$\mathrm{n}=158,1=158$

Finalmente, tenemos una muestra formada por 158 estudiantes y 23 docentes del CEBA.

Análisis de confiabilidad en la investigación

A continuación se presentan los resultados del análisis de confiabilidad referido a la Supervisión pedagógica y la 
Gestión administrativa.

Para determinar el grado de confiabilidad en los instrumentos de estudio, se aplicó el coeficiente de consistencia interna alfa de Cronbach $(\alpha)$ debido a que presentan una escala Likert.

Coeficiente Alfa de Cronbach:

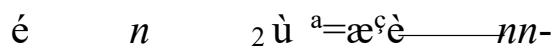

$1 \ddot{0} \div$ ๕êêê $1^{-}$ å $i=\mathrm{s} 1 \mathrm{~s} X_{2 X i}$ úúú $\quad$ ëê

úû

Donde:

n: $\mathrm{N}^{\mathrm{o}}$ de ítems

s2

$$
{ }_{x}, \mathrm{~S}^{2}: \text { Varianza }
$$

Tabla 08 Análisis de confiabilidad en la investigación

\begin{tabular}{|l|l|l|l|l|}
\hline & & & & \\
& & & & \\
& & ÍTE & Estud & Docente \\
\hline Supervisión & $158 / 23$ & $8 / 9$ & 0,634 & 0,888 \\
\hline $\begin{array}{l}\text { Pedagógica } \\
\text { Administra }\end{array}$ & $158 / 23$ & $11 / 12$ & 0,678 & 0,667 \\
t. & & & & \\
\hline
\end{tabular}


Se encontró dentro del análisis de consistencia interna "alfa de cronbach" que existe un nivel de confiabilidad aceptable en relación a la supervisión pedagógica y gestión administrativa, por lo cual, se realizará una medición objetiva de las características de interés en el presente estudio.

\section{8.- CONCLUSIONES}

8.1.- De acuerdo a los resultados observados en los estudiantes se obtuvo en la supervisión

pedagógica que el 5,7\% de los estudiantes consideran que existe una supervisión pedagógica baja, el 37.3\% un nivel medio y el 57\% considera que existe una supervisión pedagógica alta. En la gestión administrativa, que el 63.3\% de los estudiantes consideran que existe una gestión administrativa de nivel medio, el 31.6\% en un nivel alto y el 5.1\% considera que existe una gestión administrativa baja.

\section{2.-Se encontró a nivel general que existe una correlación estadísticamente} significativa $(\mathrm{P}=0.000<0.05, \mathrm{r}=0.535)$ en un nivel regular y directa entre la supervisión pedagógica y la gestión administrativa al 95\% de confianza para el caso de los estudiantes, y una correlación regular similar para el caso de los docentes $(r=0,593$, $p=0.003<0.05$ ). Por lo podemos deducir que en el caso de los estudiantes el nivel es alto de tendencia positiva, respecto al estudio de los docentes de igual forma, puesto que existe concordancia y su tendencia es media o moderada.

8.3.-A nivel de los docentes se encontró que con respecto a la supervisión pedagógica el $47.8 \%$ de los estudiantes consideran que existe una supervisión pedagógica medio, el 43.5\% un nivel alto y el $8.7 \%$ considera que existe una supervisión pedagógica bajo. Asimismo, en la gestión administrativa, el 91.3\% de los docentes consideran que existe una gestión administrativa de nivel alto, el 8.7\% en un nivel medio. 
8. 4.-Con referencia a la gestión administrativa de las TIC, los estudiantes afirman que es de nivel medio o regular, respecto a la Gestión administrativa de los docentes, pues consideran estos segundos que sí existe un nivel alto de Gestión administrativa de las TIC en el aula de Innovación pedagógica.

9. 5.-también los resultados muestran que el $41 \%$ de los trabajadores indicaron que el Nivel de uso de las TIC es de "Uso regular", el 59\% es de "Uso Alto y el "Uso bajo" de $0 \%$; en cuanto a la evaluación del nivel de desempeño muestran que el $100 \%$ de los trabajadores administrativos tienen el nivel de desempeño "Regular".

\section{9.-BIBLIOGRAFÍA}

\section{REFERENCIAS BIBLIOGRÁFICAS.}

\section{1.- Fuentes impresas}

Alvarado Oyarce, Otoniel (2002) Supervisión

Educativa (Teoría y Práctica) Lima - Perú Editora

Educativa INIDE

Arce, Alain (2000) Lima -Perú -Manual de

Supervisión Educativa- Lima Perú

Bazán León, Víctor Antonio (1997) -Monitoreo y

Supervisión Educativa - Lima Perú

Balarin, Maria. (2013) Las Políticas TIC en los Sistemas Educativos de América Latina CASO PERU. UNICEF.

Bustamante Vento, Roberto (2013) .

Coordinación del Área de Evaluación y Monitoreo Docente.

PERÚ EDUCA - Lima-Perú

Conde Curiñaupa, Regulo (2012). El aula de Innovación Pedagógica y el Aprendizaje en el área de Ciencia y Tecnología y Ambiente en el segundo grado de Educación Secundaria de la Institución Educativa Julio César Escobar de San Juan de Miraflores Lima 2012.

Cornax C.J. Del Río P.J. Sánchez A.J. (2010) La 
Supervisión del Tratamiento de las TIC, en los Centros Educativos. Servicio de Inspección de Educación de Málaga.

Chávez M. Abigail (2008). Relaciones Humanas y el Proceso de Supervisión Educativa de las Instituciones del nivel Primario de los distritos de Wánchaq y Santiago CuscoPerú. Biblioteca

Nacional del Perú.

Choque Larrauri, Raúl , (2009) . El caso de una Red Educativa de San Juan de Lurigancho de Lima (

Lima-Perú)

Directivas - DIGETE-PERÚ- EDUCA

Huascarán . html

http:// aula de

innovaciónsj.blogspot.com/2009/09/directivasdiget e-perú-educa-huascaran.html

Hernandez Sampieri, R.; Fernández, C. y Baptista, P. (2014). Metodología de la investigación. $6^{\text {a }}$ edición, México: Mc Graw Hill.

Oficina Regional de Educación para América Latina y el Caribe (OREALC/UNESCO Santiago). (Enfoques Estratégicos sobre las TIC, en educación y en América Latina)

Publicado en 2013

(Pedagogía mediada con TIC-Formación docente) Acompañamiento http://formaciondocentepedagogiacontic.blogspo t. com/p/acompanamiento-in-situ.html blog.

Octubre 2014.

Sovero Hinostroza, Franklin V. (2003) . Monitoreo y Supervisión -Editorial San Marcos Lima-Perú. 
Vigotky, (2000 p. 12) Red de Revistas

Científicas de América Latina, el Caribe, España y Portugal Sistema de Información Científica (2000)

Reflexiones sobre la escritura a partir de Vygotsky Educere, vol. 3, núm. 9, junio, 2000, Universidad de los Andes Venezuela.

\subsection{Fuentes digitales}

A.J. Juliani. (2013) Iniquiry and Innovation in the classroom. Using 20\% Time, Genius Hour, and PBL to Drive Student Success. Recuperado de http://ajjuliani.com/books/inquiryinnovationclassroom/

Ministerio de Educación -Perú (2003). Ley

General de Educación No 28044. Comisión

Permanente del Congreso de la República del

Perú. Recuperado http://www.minedu.gob.pe/p/ley_general_de_edu c acion_28044.pdf

Ministerio de Educación Perú (2009) Manual de

Supervisión Pedagógica- Área de Supervisión

Educativa..MINEDU Jr. Carabaya 650 Lima

1.Recuperado https://ugel01agp.files.wordpress.com/2011/04/ma nual-de-supervisionpedagogica-disde.pdf

Ministerio de Educación-Perú (2009) Diseño Curricular Básico Nacional de Educación Básica

Alternativa. Viceministerio de Gestión Pedagógica

Lima-Perú. Recuperado de

http://alfa.minedu.gob.pe/portal/media/2012/DCB

N_EBA_REAJUSTADO1.pdf 
Ministerio de Educación-Perú

(2010)

Lineamientos y Estrategias Generales para la

Supervisión Pedagógica. Viceministerio de Gestión

Pedagógica Lima Perú. Recuperado de

file://C:/Users/usuario/Downloads/supervision_p edagogica.pdf

Ministerio de Educación. (2010-B). Lineamientos y estrategias generales para la Supervisión Pedagógica, RVM Nº38-2009-ED. Lima:

Viceministerio de Gestión Pedagógica, Dirección de Investigación, Supervisión y

Documentación Educativa (DISDE). Viceministerio de Gestión

Pedagógica .Recuperado

http://www.asesorempresarial.com/web/adjuntossu milla/2009-08-15 TVIZXUK.pdf.

Perez de Leza Eguiguren, Javier (2000). Madrid recuperado de https://es.linkedin.com/in/javierperezdelezaeguigu ren/es

Senge et. al (1993 p.10) Peter Senge and the learning organization, recuperado de http://infed.org/mobi/peter-senge-and-thelearningorganization/

Siemens, George ( 2014). La wikiversidad -

Conectivismo $\quad, \quad$ recuperada de https://plus.google.com/100449248006461108744 /posts/55i58MrHkpT 